\title{
Efectos de las adaptaciones textuales, el conocimiento previo y las estrategias de estudio en el recuerdo, la comprensión y el aprendizaje de textos científicos
}

\author{
Eduardo Vidal-Abarca, Vicente Sanjosé, \\ JUAN JOSÉ SOLAZ \\ Universidad de Valencia
}

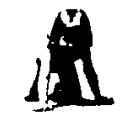

Resumen

Este estudio analiza la influencia de diversos cambios textuales, del conocimiento previo de las estrategias de estudio en el recuerdo, comprensión y aprendizaje de textos científicos. Empleamos un diseño factorial $2 \times 2$ con dos variables entre sujetos, texto y estrategias de estudio, y la variable conocimiento previo anidada bajo la variable estrategias. Universitarios de Física y Psicología, y bachilleres de ciencias y letras leyeron una de las dos versiones de un texto tomado de un libro de Física-Química de Bachillerato. En una versión se bicieron cambios para facilitar la formación del texto-base y facilitar la construcción de un modelo situacional. Se tomaron tres medidas dependientes: recuerdo, escritura de las 7-8 ideas más importantes y aprendizaje. Encontramos que las manipulaciones textuales y las estrategias de estudio produjeron diferencias significativas en todas las tareas, mientras que el conocimiento previo lo hizo en las pruebas de recuerdo y aprendizaje. Se encontraron diversas interacciones entre variables en estas dos tareas. Finalmente, comentamos algunas implicaciones de los efectos de los cambios textuales dependiendo del tipo de tarea y de las características de los estudiantes.

Palabras clave: Comprensión lectora, textos científicos, estrategias de estudio, aprendizaje mediante textos, texto-base, modelo situacional.

\section{Effects of text adaptations, previous knowledge, and study strategies on recall, comprehension, and learning from scientific texts}

\section{Abstract}

The study analyses the influence of text manipulations, previous knowledge, and study strategies on recall, comprehension, and learning from scientific texts. A $2 \times 2$ factorial design was used with two between subject variables (text and study strategies) and with a third variable (previous knowledge) nested under the strategies variable. Physics and psychology undergraduates and secondary school students, both from science and non-science options, read one of two versions of a passage from a secondary school science textbook. One of the versions had previously been changed to facilitate both the formation of a text-base and the construction of a situational model. Three dependent measures were taken: free recall, writing down the 7 or 8 most important ideas, and a learning task. Results showed that textual changes and study strategies produced significant differences on all the tasks, while previous knowledge produced these differences on recall and learning tasks. Some interactions between variables were also found in these two tasks. Finally, we discuss some implications of the effects of textual manipulations based on the type of task used and students' characteristics.

Keywords: Reading comprehension, science textbooks, study strategies, learning from texts, textbase, situational model.

Correspondencia con autores: Departamento de Psicología, Evolutiva y de la Educación. E.U. de Formación del profesorado de EGB. Universidad de Valencia. C/ Alcalde Reig, 8. 46006 Valencia.

Original recibido: Diciembre de 1992. Revisión recibida: Julio de 1993. Aceptado: Noviembre de 1993. 
De acuerdo con el modelo de Van Dijk y Kintsch (1983), a partir de la lectura de un texto, el lector puede construir dos representaciones mentales diferentes llamadas «texto-base» y «modelo situacional». El texto-base se construye a partir de las proposiciones del texto y expresa su contenido semántico tanto a nivel global como local. Esta representación mental refleja sobre todo las relaciones de coherencia entre las proposiciones así como su organización. El modelo situacional es la representación mental de la situación descrita por el texto tal como es elaborada por el lector, bien sea actualizando un modelo mental previamente existente en su mente, bien sea construyendo uno nuevo que se integre en el sistema de conocimiento del sujeto. Es decir, el modelo situacional implica una integración del contenido del texto dentro del sistema de conocimiento del lector. Tres diferencias importantes entre ambas representaciones mentales nos interesa destacar aquí. En primer lugar, el texto-base está muy influido por la coherencia global y local existente entre las proposiciones del texto debido a su estrecha conexión con el contenido semántico del texto. Sin embargo, el modelo situacional se ve influido, sobre todo, por aquellos aspectos que permiten conectar mejor el sistema de conocimiento previo del sujeto con la información textual. En segundo lugar, mientras el texto-base se evalúa mediante tareas de recuerdo libre, el modelo situacional se mide mejor por tareas de aprendizaje tales como solución de problemas, proporcionar explicaciones de hechos, realizar inferencias a partir de determinadas afirmaciones textuales y otras similares. Es decir, tareas en las que se ha de conectar la información textual con el sistema de conocimiento previo. Por último, el modelo situacional está más estrechamente relacionado con lo que normalmente llamamos «aprendizaje a partir de textos» por cuanto «el aprendizaje puede ser conceptualizado como la modificación de modelos situacionales» (Van Dijk y Kintsch, 1983, p. 342)

Naturalmente ambas representaciones no son independientes. Según Van Dijk y Kintsch, «el texto-base es una estación necesaria hacia el modelo situacional pero ambos no tienen que coincidir necesariamente» (p. 343). Hay que notar además que, según estos autores, el modelo situacional estará especialmente condicionado por una parte del texto-base, la macroestructura. Esta afirmación nos lleva a considerar otra distinción relevante para nuestro trabajo, la existente entre la micro y macroestructura del texto. Dentro del modelo al que nos estamos refiriendo, la microestructura está formada por las proposiciones que el lector va formando durante la lectura buscando estratégicamente relaciones de coherencia entre las ideas del texto. Cuando el lector recuerda un texto, recuerda estas proposiciones, si bien no todas se recuerdan igual. Las proposiciones que reflejan las ideas más importantes son mejor recordadas, al menos en el caso de los sujetos buenos lectores. Este resultado se conoce con el nombre de efecto de los niveles. La macroestructura está formada por las macroproposiciones que el lector va formando sirviéndose de macroestrategias textuales, basadas predominantemente en el texto, y de macroestrategias contextuales, basadas sobre todo en el conocimiento previo del lector. Las macroproposiciones pueden estar completamente explícitas en el texto o bien pueden estar implícitas. En el primer caso el lector únicamente debe seleccionarlas mientras que en el segundo debe producirlas. En cualquier caso, la formación de la macroestructura textual es un proceso estratégico guiado por las mencionadas macroestrategias. Una forma, entre otras, de evaluar si un lector ha formado la macroestructura textual es pedirle que escriba las ideas más relevantes de un pasaje.

Así, resumiendo lo dicho hasta aquí, vemos que el procesamiento de textos implica, según el modelo que estamos comentando, la formación de dos representaciones mentales diferentes pero relacionadas: el texto-base y el modelo 
situacional. Además, tanto en un caso como en el otro intervienen un grupo de variables relativas al lector las cuales pueden ser categorizadas en dos subgrupos, las referidas al conocimiento previo y las correspondientes a las estrategias de comprensión y estudio, y otro grupo de variables relativas al texto. Por otra parte, todas estas variables tienen una importancia diferente según el tipo de tarea que se solicita a los sujetos, entre las cuales hemos mencionado tres: recuerdo, captación de ideas principales y tareas de aprendizaje. Finalmente, estas tareas están más o menos asociadas a los dos tipos de representaciones mentales mencionadas. Estas afirmaciones tienen diverso soporte empírico que a continuación pasamos a comentar.

Por lo que se refiere a las variables textuales que afectan a la formación del texto-base, hay numerosos estudios que prueban que el recuerdo de los textos mejora cuando se incrementa la coherencia textual. Algunas de las adaptaciones textuales que incrementan la coherencia, según diversos autores (Mayer, 1985; Anderson y Armbruster, 1986; Beck y McKeown, 1989) son: introducir cambios en la organización de las ideas buscando la coherencia lógica y secuencial del tema, sustituir y/o añadir títulos para hacerlos más informativos, añadir breves resúmenes con la información más importante, destacar las ideas más importantes con claves tipográficas, añadir expresiones señal para facilitar la relación entre idea, o eliminar algunas ideas irrelevantes o muy poco importantes, entre otras varias. Las adaptaciones que mejoran el aprendizaje de los sujetos a partir de los textos, es decir, el modelo situacional, son mucho menos conocidas, si bien deben ser todas aquellas que contribuyan a conectar adecuadamente la nueva información con la existente en el sistema de conocimiento del sujeto. Así, Kintsch (1989), en una tarea de resolución de problemas aritméticos, menciona dos adaptaciones: modificaciones lingüísticas que afectan a las estructuras aritméticas subyacentes y cambios en la familiaridad de la situación representada en el problema. La inclusión de analogías en los textos también se ha mostrado eficaz para mejorar el aprendizaje a partir de textos tanto en estudiantes de primaria (Vosniadou y Schommer, 1988) como de secundaria (Dupin y Joshua, 1989). Por otra parte, la forma de presentar el contenido del mismo texto puede provocar la formación de un modelo situacional u otro. Así, Perrig y Kintsch (1985) elaboraron dos versiones de un texto que describía los lugares más importantes de una hipotética ciudad, una de las cuales tenía carácter «espacial» (tipo mapa) mientras la otra era «lineal» (tipo ruta a través de la ciudad). Se comprobó que los sujetos tendían a formar un modelo situacional acorde con la versión textual leída, si bien se encontró una interacción interesante con la variable sexo. En cualquier caso, hay que señalar que las adaptaciones textuales en los materiales que apoyan al aprendizaje académico están al servicio, lógicamente, de los objetivos centrales de la enseñanza, los cuales deberían ser el recuerdo de la información esencial y el aprendizaje, entendido como modificación de los modelos mentales previos de los sujetos, si bien hay estudios que muestran tanto la dificultad de conseguir buenas adaptaciones como de conocer las reglas acerca de cómo se pueden log rar esas buenas adaptaciones (Britton et al., 1989; Duffy et al., 1989).

Respecto a las estrategias, hay abundantes datos que confirman que los sujetos con mejores estrategias de comprensión y estudio, lo cual suele estar asociado a niveles académicos más elevados, recuerdan más información, especialmente la de más alto nivel, y además tienen un recuerdo más organizado según la estructura del texto leído (Meyer, 1984). Por lo que se refiere a la relación entre estrategias y captación de ideas principales, son bien conocidos los estudios de Brown y colaboradores en los que se muestra cómo los estudiantes de grados superiores 
ordenan las ideas de un texto según su importancia en el mismo, así como también aplican macrorreglas más complejas para seleccionar y producir las ideas más importantes (Brown y Smiley, 1978; Brown, Day y Jones, 1983). Por otra parte, Kieras (1985) aporta algunas pruebas de que sujetos universitarios parecen capaces de formar la macroestructura del texto y de captar las ideas principales de un pasaje no familiar y no comprendido en todos sus términos, basándose en un conocimiento difuso de los aspectos semánticos del texto. Así mismo, por lo que respecta al papel del conocimiento previo, es claro que los sujetos expertos en un tema, independientemente de su edad, recuerdan más que los novicios, además de tener un recuerdo mejor organizado (Chiesi, Spilich y Voss, 1979; Pearson, Hansen y Gordon, 1979; Recht y Leslie, 1988). Así mismo, cuando el conocimiento previo es activado previamente al estudio de un texto, ello mejora el recuerdo de proposiciones que presumiblemente reflejan la formación de un modelo mental adecuado, tanto en el caso de los sujetos expertos como en el de los novicios (Schmidt et al., 1989). En el caso del aprendizaje de las ciencias hay que resaltar la necesidad de partir de las concepciones previas o preconceptos de los alumnos, hacer conscientes a los estudiantes de dichas concepciones y promover el cambio conceptual para conseguir un aprendizaje efectivo, lo cual está estrechamente ligado a la consecución de un modelo situacional adecuado (p.e., Driver, 1988; Pozo, 1989).

Finalmente, por lo que respecta a las tareas que favorecen un tipo u otro de representación mental, Perrig y Kintsch (1985) constatan que sujetos que logran un buen recuerdo del texto, tarea asociada a la formación del texto-base, sin embargo, no son capaces de realizar inferencias que podrían hacer si hubieran formado un adecuado modelo situacional. Otro estudio de Schmalhofer y Glavanov (1986) proporciona datos que confirman la hipótesis de que cuando a sujetos universitarios se les pide que estudien un texto para producir un buen resumen ponen en marcha procesos de codificación y recuperación de la información que tienen carácter proposicional y son propios de la formación del texto-base. Sin embargo, cuando a un grupo similar se le pide que estudie un texto para realizar algunas actividades de aprendizaje tales como aplicar el conocimiento obtenido a la resolución de cuestiones prácticas (tarea de programación consistente en escribir y verificar expresiones de lenguaje de programación LISP), entonces los sujetos emplean procesos de codificación y recuperación de la información propios de un modelo situacional.

Estas relaciones entre variables y tareas se ha estudiado de forma separada. Además, en bastantes casos se han utilizado textos de características diferentes a los que manejan ordinariamente los estudiantes. Por ello, nuestro propósito es doble. En primer lugar tratamos de analizar el efecto conjunto de las tres variables mencionadas sobre las tareas de recuerdo, captación de ideas principales y aprendizaje utilizando un texto propio de una situación escolar. Un segundo objetivo, de carácter más aplicado, es analizar de forma más minuciosa los efectos de determinadas modificaciones sobre textos ordinarios en orden a promover la comprensión y aprendizaje de material informativo.

Así, de acuerdo con los datos expuestos previamente, planteamos cuatro hipótesis, la primera de ellas referida al papel de las variables textuales y las tres restantes relativas a la relación entre las otras variables independientemente de los aspectos textuales. En primer lugar esperamos que diversas adaptaciones textuales realizadas en orden a mejorar tanto el texto-base como el modelo situacional del lector incrementarían el recuerdo, la captación de ideas principales y el aprendizaje de los sujetos. Acerca de la interacción entre la variable texto y el resto no teníamos una hipótesis definida. En segundo lugar, formulamos la hipó- 
tesis de que los sujetos con mayor conocimiento previo y mejores macroestrategias de procesamiento obtendrían mejores resultados en tareas de recuerdo, debido al papel de ambas variables en dicha tarea. Por lo que respecta al mayor o menor peso de cada una de estas variables no teníamos tampoco una hipótesis definida. En tercer lugar, esperamos que los sujetos con mayor conocimiento previo, independientemente de sus macroestrategias, obtuvieran mejores puntuaciones en una medida de aprendizaje. Por último, en cuarto lugar enunciamos la hipótesis de que los sujetos con mejores macroestrategias obtendrían mejores resultados en una prueba de captación de ideas principales, no debiendo haber diferencias por lo que respecta al conocimiento previo.

\section{METODO}

\section{Sujetos y diseño}

Participaron en el estudio un total de 85 sujetos según la distribución que se puede ver en la Tabla I. La muestra constaba de 22 estudiantes del último curso de la Facultad de Física, 20 del último curso de la Facultad de Psicología, 20 estudiantes de $3 .^{\circ}$ de B.U.P. de la opción de Ciencias y 23 de la opción de Letras, siendo cada grupo dividido aleatoriamente en dos subgrupos aproximadamente iguales que leyeron las dos versiones del texto utilizado.

TABLA I

Distribución de los sujetos en las diferentes condiciones experimentales

\begin{tabular}{l|c|c|c|c}
\hline \multirow{2}{*}{} & \multicolumn{2}{|c|}{ Bachilleres } & \multicolumn{2}{c}{ Universitarios } \\
\cline { 2 - 5 } & Ciencias & Letras & Física & Psicología \\
\hline Texto ordinario & $10(11.8 \%)$ & $12(14.1 \%)$ & $10(11.8 \%)$ & $10(11.8 \%)$ \\
\hline Texto adaptado & $10(11.8 \%)$ & $11(12.8 \%)$ & $12(14.1 \%)$ & $10(11.8 \%)$ \\
\hline
\end{tabular}

Tal y como queda reflejado en la Tabla I, empleamos un diseño factorial $2 \times 2$ con dos variables entre-sujetos: estrategias de procesamiento (excelentes, medianas) y texto (original, adaptado) con una tercera variable, conocimiento previo, anidada en la primera de las variables, la cual constaba de cuatro grupos. Empleamos este diseño porque no es posible combinar esta última con la primera variable en un diseño factorial normal debido a que es imposible encontrar sujetos con un conocimiento previo equivalente en ciencias pero con diferente grado de dominio de estrategias de procesamiento. Así, asumimos que los sujetos del último curso de Universidad habrían de tener mejores estrategias de procesamiento que los estudiantes de Bachillerato debido a su mayor experiencia académica, si bien no realizamos ninguna prueba para confirmar nuestra suposición, aunque los mencionados datos de Brown y colaboradores, y de Kieras, entre otros, podrían avalarla. Por lo que respecta a la segunda variable, el conocimiento previo, aplicamos una prueba específica que confirmó nuestra suposición. La prueba constaba de seis preguntas abiertas que evaluaban los conocimientos previos necesarios para entender el contenido del texto que habían de leer los sujetos. Incluía unas cuestiones aplicadas sobre electrostática: a) qué ocurre cuando dos partículas del mismo y de distinto signo se encuentran próximas; b) qué ocurre cuando esa distancia se acorta; c) qué partículas son responsables de la carga eléctrica de los cuerpos; d) cómo se puede explicar un fenómeno de electrificación 
por frotamiento; e) qué trayectoria siguen distintas partículas positivas lanzadas sobre otra partícula también positiva. Los contenidos de electrostática son previos al estudio del tema de Modelos Atómicos, que fue el escogido para nuestro estudio. Dichos contenidos eran necesarios para comprender tanto el experimento de Rutherford como su modelo atómico, todo lo cual era el contenido central del texto que se había de leer. Encontramos diferencias significativas en la prueba entre los universitarios de Física (UF) y el resto de los grupos ( $\mathrm{p}<.01)$. Así mismo, había diferencias significativas entre los bachilleres de ciencias (BC) y los dos grupos restantes $(\mathrm{p}<.05)$, bachilleres de letras $(\mathrm{BL})$ y universitarios de Psicología (UP), no habiéndolas entre estos dos últimos subgrupos. La tercera de las variables, el texto, fue la única manipulada en nuestro experimento. A continuación pasamos a comentar las modificaciones textuales realizadas.

\section{Materiales}

El texto empleado pertenecía al tema de Modelos Atómicos de un libro de Física-Química de 2. ${ }^{\circ}$ de B.U.P., por lo cual era de un contenido previamente conocido por todos los sujetos. El texto tenía dos partes. En la primera se explicaba la evolución de los modelos atómicos (modelos de Dalton, Thomson y Rutherford), mientras en la segunda se hablaba sobre la composición del núcleo atómico (partículas subatómicas: protón y neutrón). Naturalmente, los estudiantes de ciencias habían de tener mejor conocimiento de su contenido que los de letras. Por lo que respecta a estos últimos, los bachilleres deberían tener más reciente su contenido mientras que los universitarios lo habrían de tener bastante más olvidado, al menos en sus detalles precisos. Tomamos la mayor parte del contenido del tema (texto, figuras y ejemplos) dejando aparte las cuestiones y problemas. Escogimos dicho tema porque en el mismo predominaba la exposición de información textual y contaba con pocas formulaciones físico-matemáticas. Del mismo texto elaboramos dos versiones. La ordinaria era idéntica al texto original pero estaba reescrita para evitar diferencias en tipografía, contando con un total de 1.600 palabras aproximadamente. En la versión adaptada introdujimos diversas modificaciones que a continuación pasamos a comentar. Esta versión era un poco más extensa, teniendo alrededor de 1.900 palabras. Las modificaciones introducidas en el texto se pueden agrupar en dos categorías: aquellas que pretendían mejorar la coherencia textual con el fin de facilitar y mejorar la formación del texto-base, y aquellas otras que pretendían promover una mejor formación del modelo situacional por parte de los sujetos. Estas últimas estaban dirigidas a favorecer la integración del texto dentro de los conocimientos previos del lector. A continuación pasamos a enumerar ambos tipos de modificaciones.

A. Modificaciones para mejorar la coherencia textual:

1. Cambios en la organización de las ideas buscando el desarrollo lógico y secuencial del contenido.

2. Sustitución y/o adición de títulos para hacerlos más informativos.

3. Adición de breves resúmenes con la información más importante.

4. Señalamiento de las ideas más importantes con claves tipográficas.

5. Colocación en párrafos distintos de la información temáticamente diferente.

6. Adición de expresiones señal para facilitar la relación entre ideas.

7. Reducción de la complejidad sintáctica de algunas frases.

8. Redundancia con la información importante o problemática.

9. Eliminación de algunas ideas irrelevantes o muy poco importantes. 
B. Modificaciones para mejorar la formación del modelo mental de los sujetos:

1. Inserción de analogías que proporcionaran una imagen mental adecuada de algunas informaciones del tema (p.e., el modelo atómico de Thomson ha sido denominado como «modelo de tarta de pasas»; o bien, «el modelo atómico de Rutberford ha sido llamado modelo planetario») .

2. Incluir algunas ideas presumiblemente conocidas previamente por los sujetos de forma que se redujera la necesidad de inferencias en algunas informaciones importantes del texto (p.e., la materia está compuesta de átomos; las cargas eléctricas positivas neutralizan a las cargas negativas).

3. Inserción de preguntas para activar los conocimientos previos de los sujetos referidos a información esencial que el texto comunicaba (p.e., Nos podemos preguntar a partir de nuestros conocimientos en electrostática cómo puede ser estable un agrupamiento de protones en una región tan pequeña como es el núcleo atómico, a pesar de ser partículas cargadas positivamente y, como tales, se repelen. Nos podemos preguntar también por qué sumando las masas de protones y electrones para un determinado átomo, no obtenemos la masa atómica).

4. Enfatizar conceptos que contradicen las concepciones espontáneas o preconceptos de los alumnos (p.e., en relación con la dificultad en admitir la existencia de «espacio vacío»: En este modelo (Rutherford) se supone al átomo constituido por un núcleo central diminuto, de gran masa y cargado positivamente, y por unos electrones moviéndose alrededor del núcleo en un gran volumen vacío). El texto ordinario mencionaba superficialmente algunos de estos conceptos, mientras que el texto adaptado aludía a ellos en diversas ocasiones y al comienzo o final de párrafo para resaltar su importancia.

Junto a estas modificaciones textuales, efectuamos algunas otras en las tres figuras que contenía el texto original. Todas ellas fueron dibujadas de una forma un poco más esquemática. Además, en la versión adaptada añadimos una cuarta figura para representar el modelo atómico de Rutherford, cuyo conocimiento era uno de los contenidos centrales del texto. Hay que advertir que las modificaciones efectuadas afectaron especialmente a los contenidos que se consideraron importantes.

\section{Medidas y puntuación}

Se tomaron tres medidas dependientes. La primera fue una prueba de recuerdo. Dada la gran extensión del texto optamos por dividirlo en ideas-unidad amplias (p.e., idea 13: "Rutherford observó en su experimento que la mayoría de las partículas atravesaban la lámina de oro, algunas sufrían una pequeña desviación y unas pocas retrocedian»). Seguimos para ello un procedimiento similar al empleado por García Madruga y Martín Cordero (1987). Dichas ideas unidad fueron categorizadas en dos niveles: «micro» y «macro» de acuerdo con su importancia dentro del texto, obteniendo 18 ideas de micronivel y 17 de macronivel. Otorgamos 1 punto a las primeras y 2 puntos a las segundas, restando la mitad de cada uno de los valores respectivos en caso de que la idea no estuviese completa (p.e., "Rutherford observó en su experimento que algunas partículas se desviaban»). Los protocolos de recuerdo fueron corregidos separadamente por dos de los autores del trabajo, obtenido un acuerdo entre jueces de $87 \%$, resolviendo las discrepancias de común acuerdo.

En la segunda medida, captación de ideas principales, se definieron las ocho ideas más importantes que contenía el texto a juicio de los autores de este trabajo, entendiendo por tales aquella información esencial que el autor del texto 
intentaba transmitir, la cual sería la información básica que cualquier persona con una cultura de bachiller debería conocer aunque no continuara estudiando ciencias. Seis de las ideas eran más complejas, siendo puntuadas con 2 puntos si estaban completas, con 1 punto si les faltaba algún elemento importante y con 0 en cualquier otro caso. Así, por ejemplo, la idea principal 4 era: «La masa y la carga positiva del átomo se concentran en el núcleo; la carga negativa envuelve a éste». Si los sujetos aludían sólo a las ideas referidas a la «carga eléctrica» pero no a la relativa a la «masa» se otorgaba 1 punto. Las dos ideas restantes eran puntuadas con 0 ó 1 . Los protocolos de los sujetos fueron puntuados independientemente por dos de los autores del trabajo en orden a valorar la presencia de las ideas mencionadas, obteniendo un porcentaje de acuerdos de $89 \%$.

La tercera medida evaluaba la capacidad de los sujetos para aplicar los conocimientos adquiridos a contextos o situaciones nuevas. Es decir, esta medida estaba más próxima que las restantes a lo que definimos en la introducción como «aprendizaje a partir de textos». Es decir, supone una modificación de esquemas a partir de la conexión entre la información nueva y la que el sujeto tenía previamente. Por ello, en lo sucesivo nos referiremos a ella como la prueba de aprendizaje, aunque las restantes también evalúan otros tipos de aprendizaje (una discusión sobre el concepto de aprendizaje se puede ver en el libro de Pozo, 1989, y en Vidal-Abarca, 1992, entre otros trabajos). Constaba de cinco ítems de respuesta abierta, tres ellos de resolución de problemas o cuestiones (sólo uno de ellos implicaba la aplicación de un algoritmo inferible claramente a partir de la información explicada en el texto), siendo los dos restantes preguntas en las que había que razonar algunas afirmaciones o hechos experimentales para lo cual era necesario relacionar diversas ideas textuales. Cada uno de los ítems fue puntuado como correcto (1 punto) o incorrecto ( 0 puntos). El acuerdo entre jueces fue del $94 \%$. Dos muestras de los ítems empleados en la prueba son los siguientes:

Item 1. Si en el experimento de Rutherford se bubieran utilizadoátomos cargados negativamentecomo proyectiles, y los resultados obtenidos hubieran sido los mismos, ¿ qué modelo propondrías para el átomo?

Item 2. ¿A quése debe que unas partículas positivas (los proyectiles) se desvien más que otras en la experiencia de Rutherford?

\section{Procedimiento}

La aplicación de las pruebas se llevó a cabo a lo largo de dos sesiones de una hora aproximada de duración cada una, siendo aplicadas de forma colectiva en el centro de estudio de cada uno de los sujetos. En la primera de las sesiones se explicó la finalidad de la investigación y se aplicó la prueba de conocimiento previo. A continuación se repartió de forma aleatoria entre los sujetos el texto sobre Modelos Atómicos en sus dos versiones. Se dijo a los estudiantes que tenían que estudiarlo durante 25 minutos, advirtiéndoles que posteriormente deberían escribir toda la información que recordaran. Al terminar el tiempo estipulado, y tras un breve período de descanso, pudieron escribir toda la información que recordaban durante 20 minutos.

La segunda sesión tuvo lugar una semana más tarde. En ella se instruyó brevemente a los sujetos con un corto texto expositivo acerca de la tarea de escribir las ideas principales de un pasaje. Mostramos cómo las ideas principales podían diferir en su grado de generalidad, complejidad sintáctica y elaboración, pero deberían pertenecer al contenido del texto y estar formuladas en forma de oración (p.e., un tema o tópico no sería válido). Posteriormente se les entregó un ejemplar del texto en la misma versión que habían leído previamente, pidiéndoseles que, durante 15 minutos, escribieran las 7-8 ideas más importantes que, a 
su juicio, el autor intentaba transmitir a los lectores. Por último se repartió la prueba de aprendizaje, la cual pudieron contestar durante 20 minutos.

\section{RESULTADOS}

De acuerdo con el diseño mencionado anteriormente, los datos de cada una de las medidas fueron analizados mediante diversos ANOVAs tomando como variables dependientes las siguientes: estrategias de procesamiento, texto y conocimiento previo, estando esta última anidada en la primera. Los correspondientes a la prueba de recuerdo se pueden ver en la Tabla II.

TABLA II

Media y (desviacione estándar) de los sujetos de los diferentes grupos en las medias de recuerdo (T.A.: Texto adaptado; T.O.: Texto ordinario

\begin{tabular}{|c|c|c|c|c|c|c|c|c|}
\hline \multirow{5}{*}{$\begin{array}{l}\text { TOTAL } \\
\text { MACRO } \\
\text { MICRO }\end{array}$} & \multicolumn{4}{|c|}{ Bachilleres } & \multicolumn{4}{|c|}{ Universitarios } \\
\hline & \multicolumn{2}{|c|}{ Ciencias } & \multicolumn{2}{|c|}{ Letras } & \multicolumn{2}{|c|}{ Física } & \multicolumn{2}{|c|}{ Psicología } \\
\hline & 9.7 & $(3.98)$ & 8.7 & $(3.42)$ & 23.41 & $(5.80)$ & 14.30 & (6.70) \\
\hline & 8.15 & $(3.96))$ & 7.26 & (2.68) & 18.32 & (4.84) & 11.65 & (5.80) \\
\hline & 1.50 & (1.38) & 1.43 & $(1.25)$ & 5.02 & (1.80) & 2.65 & (1.39) \\
\hline & T.A. & T.O. & T.A. & T.O. & T.A. & T.O. & T.A. & T.O. \\
\hline TOTAL & 12.5 & 6.8 & 10.8 & 6.7 & 27.1 & 18.9 & 17.2 & 11.4 \\
\hline & (2.8) & (2.6) & $(3.1)$ & $(2.4)$ & $(4.5)$ & (3.6) & $(7.3)$ & (4.8) \\
\hline MACRO & 10.9 & 5.4 & 8.8 & 5.8 & 21.6 & 14.4 & 14.3 & 9.0 \\
\hline & (2.4) & (3.2) & (2.8) & (1.7) & (3.4) & (3.1) & $(6.2)$ & (4.1) \\
\hline MICRO & 1.6 & 1.4 & 2.0 & 0.9 & 5.5 & 4.5 & 2.9 & 2.4 \\
\hline & (1.0) & (1.7) & (1.4) & (0.9) & $(2.2)$ & (0.8) & (1.8) & (0.8) \\
\hline
\end{tabular}

Por lo que respecta al recuerdo total de ideas, encontramos diferencias significativas en las variables principales, Estrategia, $\mathrm{F}(1,80)=109.503, \mathrm{p}<.001$, Texto $\mathrm{F}(1,80)=45.332, \mathrm{p}<.001$, y Conocimiento previo dentro del grupo con excelentes estrategias, $\mathrm{F}(1,80)=47.242, \mathrm{p}<.001$, y puntuando más alto los universitarios, aquellos que leyeron el texto adaptado y los universitarios de Física, respectivamente. Además, realizados diversos análisis post-hoc mediante el test de Scheffe, encontramos que el grupo UF puntuó más alto que el resto de los grupos $(\mathrm{p}<.01)$. A continuación, la puntuación más alta fue la de los universitarios de Psicología (UP), los cuales puntuaron significativamente más alto que los dos grupos de bachilleres (BC y BL) $(\mathrm{p}<.01)$, no habiendo diferencias significativas entre estos dos últimos grupos. Hay que mencionar igualmente que los sujetos de cada uno de los cuatro grupos (UF, UP, BC, BL) que leyeron la versión adaptada puntuaron significativamente mejor que aquellos que leyeron la versión original $(\mathrm{p}<.05)$. Los datos correspondientes al recuerdo de ideas de nivel «macro» son prácticamente idénticos a los que acabamos de exponer con la única excepción de que, si bien la puntuación del grupo BL con el texto adaptado fue superior a la de aquellos que leyeron la versión original, en este único caso las diferencias no fueron significativas. Por lo que respecta al recuerdo de ideas de nivel «micro», los resultados se alejan algo más de los expuestos para la puntuación total de recuerdo. Así, si bien la variable Texto globalmente considerada produce diferencias significativas $(\mathrm{p}<.05)$, en las pruebas post-hoc encontramos que nin- 
guno de los cuatro grupos de sujetos puntuó significativamente mejor con la versión adaptada que con la original.

Los datos de la prueba de captación de ideas principales se pueden ver en la Tabla III. Se observaron diferencias significativas únicamente en las variables Estrategia, $\mathrm{F}(1,80)=4,544, \mathrm{p}<.05$ y Texto, $\mathrm{F}(1,80)=68.922$, $\mathrm{p}<.001$, puntuando los universitarios mejor que los bachilleres, y los que leyeron el texto adaptado mejor que los que leyeron la versión original. Hay que notar que en los análisis post-hoc hallamos que las puntuaciones de los grupos UF y UP fueron muy similares entre sí y superiores a las de ambos grupos de bachilleres (BC y BL), si bien no lo fueron al nivel de significación usualmente admitido. Sin embargo, encontramos que los dos grupos de universitarios (UF y UP) que leyeron la versión adaptada puntuaron significativamente mejor que los grupos correspondientes que leyeron la versión original $(\mathrm{p}<.05)$, lo cual no ocurrió en el caso de los bachilleres, aunque también éstos obtuvieron puntuaciones más elevadas con la versión adaptada que con la original.

TABLA III

Mediay (desviaciones estándar) de los sujetos de los diferentes grupos en la prueba de captación de ideas principales $(T \cdot A .=$ Texto adaptado; $T \cdot O .=$ Texto ordinario $)$.

\begin{tabular}{l|c|c|c|c|c|c|c|c}
\hline \multirow{2}{*}{} & \multicolumn{4}{|c|}{ Bachilleres } & \multicolumn{4}{c}{ Universitarios } \\
\cline { 2 - 9 } & \multicolumn{2}{|c|}{ Ciencias } & \multicolumn{2}{c|}{ Letras } & \multicolumn{2}{c}{ Física } & \multicolumn{2}{c}{ Psicología } \\
\hline TOTAL & 4.25 & $(1.77)$ & 4.35 & $(1.64)$ & 5.46 & $(2.81)$ & 5.30 & $(2.99)$ \\
\hline & T.A. & T.O. & T.A. & T.O. & T.A. & T.O. & T.A. & T.O. \\
\hline TOTAL & $\begin{array}{c}5.7 \\
(1.6)\end{array}$ & $\begin{array}{c}3.5 \\
(0.9)\end{array}$ & $\begin{array}{c}5.3 \\
(1.8)\end{array}$ & $\begin{array}{c}3.8 \\
(1.6)\end{array}$ & $\begin{array}{c}7.0 \\
(3.2)\end{array}$ & $\begin{array}{c}4.2 \\
(2.0)\end{array}$ & $\begin{array}{c}7.0 \\
(3.8)\end{array}$ & $\begin{array}{c}4.2 \\
(1.6)\end{array}$ \\
\hline
\end{tabular}

Los datos de la prueba de aprendizaje pueden verse en la Tabla IV. Encontramos diferencias significativas en las variables Estrategias, $\mathrm{F}(1,80)=7.878, \mathrm{p}<.01$, Texto, $\mathrm{F}(1,80)=13.018, \mathrm{p}<.001$, y los grupos de conocimiento previo, tanto los anidados dentro del nivel medio de estrategias (bachilleres), $\mathrm{F}(1,80)=3.964, \mathrm{p}<.05$, como los anidados dentro del nivel excelente (universitarios), $\mathrm{F}(1,80)=26.010, \mathrm{p}<.001$. Así, puntuaron más alto los grupos siguientes: estudiantes que leyeron la versión adaptada, universitarios y ambos grupos de estudiantes de ciencias comparados con sus equivalentes en letras, respectivamente. Así mismo, es significativa la interacción entre Estrategias y Texto, $\mathrm{F}(1,80)=5.765$, $\mathrm{p}<.05$ debido a que los bachilleres, especialmente los de ciencias, mejoraron enormemente con el texto adaptado mientras que esto no surtió apenas ningún efecto en el caso de los universitarios. De hecho, en las pruebas post-hoc únicamente se apreciaron diferencias entre los sujetos que leyeron ambas versiones en el caso de los BC $(\mathrm{p}<.01)$ y los $\mathrm{BL}(\mathrm{p}<.05)$. Además, hay que resaltar que el efecto positivo de las manipulaciones textuales en el grupo BC fue tan grande, que aquellos que leyeron la versión adaptada puntuaron significativamente más alto que el resto de los grupos, a excepción obviamente de UF, con el cual redujeron diferencias espectacularmente. Sin embargo, no existen diferencias en los grupos de universitarios que leyeron ambas versiones textuales, si bien las razones son previsiblemente las opuestas en ambos grupos. Así, los UF te-nían tanto conocimiento previo que las modificaciones textuales les resultaron poco relevantes para esta tarea, mientras que ese 
mismo resultado se debió previsiblemente al bajo conocimiento previo en el caso de los UP.

TABLA IV

Media y (desviaciones estándar) de los sujetos de los diferentes grupos en la prueba de aprendzaje (T.A. = Texto adaptado; T.O. $=$ Texto ordinario) .

\begin{tabular}{|c|c|c|c|c|c|c|c|c|}
\hline \multirow[b]{3}{*}{ TOTAL } & \multicolumn{4}{|c|}{ Bachilleres } & \multicolumn{4}{|c|}{ Universitarios } \\
\hline & \multicolumn{2}{|c|}{ Ciencias } & \multicolumn{2}{|c|}{ Letras } & \multicolumn{2}{|c|}{ Física } & \multicolumn{2}{|c|}{ Psicología } \\
\hline & 2.75 & (1.80) & 1.91 & $(1.20)$ & 4.27 & (1.08) & 2.1 & (1.61) \\
\hline & T.A. & T.O. & T.A. & T.O. & T.A. & T.O. & T.A. & T.O. \\
\hline TOTAL & $\begin{array}{c}3.8 \\
(1.8)\end{array}$ & $\begin{array}{c}1.7 \\
(1.1)\end{array}$ & $\begin{array}{c}2.6 \\
(1.4)\end{array}$ & $\begin{array}{c}1.3 \\
(0.5)\end{array}$ & $\begin{array}{c}4.4 \\
(1.2)\end{array}$ & $\begin{array}{c}4.1 \\
(1.0)\end{array}$ & $\begin{array}{c}2.3 \\
(1.9)\end{array}$ & $\begin{array}{c}1.9 \\
(1.4)\end{array}$ \\
\hline
\end{tabular}

\section{CONCLUSIONES}

Resumiendo los principales resultados de nuestro estudio, encontramos que el efecto de las tres variables que hemos considerado, estrategia de estudio y comprensión, conocimiento previo y adaptaciones textuales, así como el ocasionado por las diferentes combinaciones entre ellas, es sustancialmente diferente en las distintas tareas planteadas a los sujetos. A continuación pasamos a revisar pormenorizadamente cada una de las hipótesis planteadas en el estudio.

Nuestra primera hipótesis predecía que las diversas adaptaciones textuales incrementarían el recuerdo, la captación de ideas principales y el aprendizaje de los sujetos. La hipótesis se confirma ya que la variable texto es significativa en todas las medidas. Sin embargo se produjeron interacciones interesantes, sobre las que no teníamos una hipótesis previamente definida, las cuales pasamos a comentar. En primer lugar, casi todos los subgrupos de estudiantes (BC, UF y UP) mejoraron significativamente en el recuerdo de las ideas de nivel «macro» cuando leyeron el texto adaptado pero no ocurrió otro tanto cuando recordaron las de nivel «micro». La razón creemos que está en que, tal como dijimos, las modificaciones textuales recayeron fundamentalmente en el primer tipo de ideas. En segundo lugar, por lo que respecta a la tarea de captación de ideas principales, las modificaciones textuales afectaron positivamente de forma significativa únicamente a los dos grupos de universitarios. Este dato se podría explicar si consideramos otros estudios que muestran que las tareas que demandan algún tipo de diferenciación precisa de niveles de importancia o de producción de las ideas importantes de un pasaje resultan particularmente complejas (Brown y Smiley, 1978; Brown y Day, 1983). Parece que las modificaciones textuales únicamente pueden ser bien aprovechadas por sujetos que tengan unas buenas estrategias de comprensión y estudio. En tercer lugar, y de forma opuesta a lo que acabamos de resaltar, las modificaciones textuales afectaron positivamente de forma significativa únicamente a los dos grupos de bachilleres, especialmente a los de ciencias, en la prueba que mide el aprendizaje a partir del texto. Pensamos que ello se debe sobre todo a aquellas adaptaciones textuales que estaban orientadas a mejorar el modelo mental de los sujetos. Dichas adaptaciones lograrían activar los conocimientos previos así como conectar adecuadamente la nueva información con la que ya tenían previamente los estudiantes. Este resultado es congruente con el obtenido por Schmidt et al. (1989) que expusimos en la introducción, referido a que la activación de los conocimientos previos previa a la lec- 
tura de un texto incide positivamente en la formación del modelo mental adecuado tras la lectura del mismo, así como a los trabajos que aludían al efecto positivo de la inclusión de analogías (Vosniadou y Schommer, 1988; Dupin y Joshua, 1989). Además, este dato contrasta con el que acabamos de exponer referido al recuerdo de proposiciones de nivel macro. Mientras que el incremento de coherencia propio del texto adaptado mejora el recuerdo de todos los grupos, el segundo tipo de modificaciones textuales sólo actúa sobre aquellos sujetos que tienen un nivel de conocimiento previo tal que permite ser activado, es decir, los bachilleres, especialmente los de ciencias. Sin embargo, las modificaciones textuales no tienen efecto alguno en los universitarios debido, o bien a que su conocimiento previo no necesita ser activado (UF), o bien a que es particularmente bajo (UP).

La segunda hipótesis predecía que los sujetos con mayor conocimiento previo y mejores estrategias de comprensión y estudio obtendrían mejores resultados en la tarea de recuerdo, independientemente de la variable texto, debido al papel de ambas variables en dicha tarea. Esta hipótesis también se confirma por cuanto ambas variables, conocimiento previo y estrategias, son significativas, obteniendo, además, el grupo de universitarios de ciencias resultados significativamente mejores que el resto de los grupos. Como se recordará, no teníamos una hipótesis definida acerca de la interacción entre ambas variables. Los resultados obtenidos aclaran el sentido de la misma. Así, hemos encontrado que los universitarios de Psicología puntúan significativamente mejor que los dos grupos de bachilleres. Además, no hemos encontrado diferencias significativas entre estos dos últimos grupos. Todo ello parece apuntar a que el recuerdo es un producto de la interacción del conocimiento previo y de las estrategias de estudio, si bien estas últimas tienen un papel más relevante, al menos cuando todos los sujetos tienen un mínimo conocimiento del tema. Estos resultados son, en apariencia, parcialmente contradictorios con los de otros autores mencionados en la introducción (Chiesi, Spilich y Voss, 1979; Pearson, Hansen y Gordon, 1979; Recht y Leslie, 1988). Creemos que esta contradicción se puede explicar por cuanto, en nuestro caso, los sujetos del grupo BC no eran realmente expertos, así como los grupos BL y UP tampoco eran completamente novicios. Es decir, parece que cuando existen niveles intermedios más y menos elevados de conocimiento previo, son más relevantes las estrategias de estudio y comprensión que el conocimiento previo.

La tercera de nuestras hipótesis predecía que los sujetos con mayor conocimiento previo, independientemente de sus estrategias y del texto leído, obtendrían mejores puntuaciones en la medida de aprendizaje. Esta hipótesis no se confirma totalmente. Así, sólo uno de los grupos de ciencias (UF) tiene puntuaciones significativamente mejores que el resto, pero no ocurre otro tanto con el otro grupo (BC). Este resultado hemos de ponerlo en relación con el mencionado en la primera hipótesis. Allí aludíamos a una interacción entre las tres variables empleadas en nuestro estudio ya que el grupo BC únicamente alcanza puntuaciones en esta medida de aprendizaje cuando leen el texto adaptado. Es decir, parece que el conocimiento previo podría ser el más relevante para resolver adecuadamente esta tarea, pero sólo cuando el texto, u otros procedimiento previos al estudio, consiguen activar los conocimientos previos necesarios y/o favorecer un modelo situacional adecuado.

Por último, la cuarta hipótesis predecía que los sujetos con mejores estrategias de comprensión y estudio obtendrían mejores resultados en la prueba de captación de ideas principales, no debiendo haber diferencias por lo que respecta al conocimiento previo. Esta hipótesis se confirma parcialmente. Efectivamente, 
la variable Estrategia produce diferencias significativas, no ocurriendo lo mismo con la variable conocimiento previo. Además, la puntuación de los universitarios de Física es prácticamente idéntica a la de los de Psicología. No obstante, si bien estos dos grupos puntúan más alto que los bachilleres, no alcanzan un nivel significativo de mejora. Todos estos resultados son congruentes con los mencionados por Kieras (1985), a los que aludimos en la primera parte del estudio, en el sentido de que los sujetos universitarios parecen capaces de captar las ideas principales de un pasaje no familiar y no comprendido en todos sus términos, basándose en un conocimiento difuso de los aspectos semánticos del texto.

Nuestro estudio creemos que aporta mayor claridad a las relaciones que se establecen entre los tres grupos de variables mencionados. Así mismo, confirmamos la distinción del modelo de Van Dijk y Kintsch (1983) y de investigaciones posteriores (Perrig y Kintsch, 1985; Schmalhofer y Glavanov, 1986) acerca de la diferenciación entre los dos tipos de representaciones mentales que las personas formamos tras la lectura y estudio de un texto dependiendo su mayor o menor adecuación de variables textuales, personales o de tarea. Igualmente creemos que del mismo se derivan algunas repercusiones acerca de la mejora de los libros de textos. No obstante quedan diversas cuestiones que no están suficientemente clarificadas y requieren posterior investigación. En primer lugar, es necesario delimitar más claramente los efectos de los dos tipos de modificaciones textuales realizadas, así como las variables textuales que han de ser manipuladas, especialmente por lo que respecta a aquellas que pretenden mejorar el modelo mental del sujeto. En segundo lugar, es preciso obtener medidas mucho más precisas de la variable Estrategias de comprensión y estudio, diferenciando entre ellas y aislándolas de otras como el conocimiento previo. Además, y desarrollando un poco más esta última observación, es preciso diferenciar entre estrategias más dirigidas al aprendizaje y aquellas otras enfocadas preferentemente al recuerdo. Por último, se necesitan procedimientos para medir de forma más precisa el conocimiento previo de los sujetos, tarea en la que ya hay intentos muy interesantes (p.e., Goldsmith y otros, 1991). Una vez que estos tres grupos de variables puedan ser medidos de forma más precisa será posible abordar en mejores condiciones la relación entre todos ellos en las distintas tareas propias del estudio y el aprendizaje a partir de textos.

\section{Referencias}

Anderson, T. H., y Armbruster, B. B. (1986). Readable textbook or selecting a textbook is not like buying a pair of shoes. En J. Orasanu (Ed.), Reading comprehension: from research to practice. Hillsdale, N.J., Lawrence Erlbaum Associates.

Beck, I. L. y McKeoown, M. G. (1989). Expository texts for young readers. En L. B. Resnick (Ed.), Knowing, learning and instruction. Hillsdale, N.J., Lawrence Erlbaum Associates.

Britton, B. K.; VAN Dussen L.; Gulgoz, S., y GLYNN, S. M. (1989). Instructional texts rewriten by five expert teams: revision and retention improvements. Journal of Educational Psychology, 81, 2, 226239.

Brown, A. L., y Smiley, S. S. (1978). The development of strategies for studying texts. Child Development, 49, 1.076-1.088.

Brown, A. L.; DAY J. D., y Jones, R. S. (1983). The development of plans for summarizing texts. Chil Development, 54, 968-979.

Chiesi, H. L.; SpIlich, G. J., y Voss, J. F. (1979). Adquisition of domain-relat ed information in relation to high- and low-domain knowledge. Journal of Verbal Learning and Verbal Behavior, 18, 257-274.

Driver, R. (1988). Un enfoque constructivista para el desarrollo del currículo en ciencias. Enseñanza de las ciencias, 6, 109-120. 
Duffy, T. M.; Higgins, L.; Mehlenbacher, B.; Cochram, C.; Wallace, D.; Hill, C.; Haugen, D.; McCaffrey, M.; Burnett, R.; SloAne, S., y SMith, S. (1989). Models for the design of instructional text. Reading Research Quarterly, XXIV, 4, 434-457.

Duppin, J.J., Y JoshuA, S. (1989). Analogies and modeling analogies in teaching: some examples in basic electricity. Science Education, 73, 207-224.

García Madruga, J.A., y Martín Cordero, J. (1987). Aprendizaje, comprensión y retención de textos. Madrid, U.N.E.D.

Goldsmith, T. E.; Johnson, P. J., у Acton, W. H. (1991). Assesing structual knowledge. Journal of Educational Psychology, 83, 1, 88-96

Kieras, D.E. (1985). Thematic Processes in the Comprehension of Technical Prose. En B. K. Britton y J. B. Black: Understanding Expository Text Hillsdale, N. J., Lawrence Erlbaum Associates.

KINTSCH, W. (1989). Learning from texts. En L. B. Resnick (Ed.), Knowing, learning and instruction. Hillsdale, N. J., Lawrence Erlbaum Associates.

MAYer, R.E. (1985). «Structural Analysis of Science Prose: Can We Increase Problem-Solving Performance?» En B. K. Britton y J. B. Black: Understanding Expository Text. Hillsdale, N. J., Lawrence Erlbaum Associates.

Meyer, B. J. F. (1984). Text dimensions and cognitive processing. En H. Mandl, N. L. Stein y T. Trabasso (Eds.), Learning and comprehension of text. Hillsdale, N.J. Lawrence Erlbaum Associates.

Pearson, P.D.; Hansen, J., y Gordon, C. (1979). The effect of background knowledge on young children's comprehension of explicit and implicit information. Journal of Reading Behavior, 11, 201-209.

Perrig, W., y KInTsch, W. (1985). Propositional and situational representations of text. Journal of Memory and Language, 24, 503-518.

Pozo, J.I. (1989). Teorías cognitivas del aprendizaje. Madrid, Morata.

RECHT D.E., y LESLIE L. (1988). Effect of prior knowledge on good and poor readers' memory of text. Journal of Educational Psychology, 80, 1, 16-20.

Schmalhofer, F., y Glavanov, D. (1986). Three components of understanding a programmer's manual: verbatim, propositional, and situational representati ons. Journal of Memory and Language, 25, 279-294.

Schmidt, H.G.; De Volder, M.L; De Grave, WS., Moust, J.H.C., y Patel, V.L. (1989). Explanatory models in the processing of science text: the role of prior knowledge activation through small-group discussion. Journal of Educational Psychology, 81, 4, 610-619.

VAN DiJK, T., y KINTSCH, W. (1983). Strategies of Discourse comprehension. Nueva York: Academic Press.

Vidal-Abarca, E. (1992). Psicología Evolutiva y de la Educación en la formación de profesores. Proyecto docente de Cátedra de Escuela Universitaria. Universidad de Valencia.

VosANIADOU, S., y SCHOMMER, M. (1988). Explanatory analogies can helpchildre n acquiere information from expository text. Journal of Educational Psychology, 80, 4, 524-536.

\section{Extended Summary}

According to van Dijk and Kintsch's (1983) model, after reading a text, readers may construct two different mental representations which they call "text-base" and "situational model". There are three important differences between both types of representations which we would like to point out. First, while the text-base is strongly influenced by the propositions' local and global coherence, the situational model is especially affected by textual variables which enable the reader's previous knowledge to connect with available text information. Secondly, while the text-base is assessed through tasks involving free recall or understanding the main ideas in a text, the situational model is better assessed through tasks which involve applying text information to new contexts. Thirdly, the situational model is more closely related to what is known as "learning from texts" than textbase. In addition, the situational model will be particularly conditioned by the text's macrostructure, a part of the textbase. Macrostructure formation is guided by strategic processes known as macrostrategies, which may be assessed through tasks such as writing the most important ideas in a text.

In summary, the study's theoretical framework supports that after reading a text, expert readers may construct two different but related mental representations, influenced by different text variables. Likewise, a reader's 
previous knowledge and macrostrategies differentially influence the construction of both mental representations. Finally, it seems that different study tasks, carried out by subjects after reading a text, are more representative of one or the other type of mental representation.

In agreement with the present model, the following four hypotheses are put forth. One, we expected that various text adaptations would improve recall, understanding the main ideas in a text, and learning. Two, we predicted that previous knowledge and macrostrategies would play a positive role on recall, though we had no clear prediction on the relative importance of these two variables. Three, we expected that subjects who had greater prior knowledge, independent from their macrostrateg ies, would obtain better results in learning tasks. Finally, we predicted that, independent of prior knowledge, those readers with better macrostrategies would obtain better results than readers with poorer strategies in a task on understading the main ideas in a text.

To study these hypotheses, we used a $2 \times 2$ factor design with two between subject variables — text (original and adapted) — and processing strategies - (excellent and moderate)_ with a third variable — previous knowledge- nested under the strategies variable. According to the design, 85 subjects participated in the study, distributed into four groups: science secondary school students (SS group), non-science secondary school students (SnS group), physics undergraduates (Uph), and psychology undergraduates (Ups group). Approximately half the students from each group read the origal version of a text and the other half an adapted version.

We selected a large passage from a science textbook for 15-16 yr. olds on atomic models. The passage had two different versions, the original and an adapted form. In the latter two types of modifications were introduced. First, several changes were made in the text in order to improve text coherence and facilitate text-base formation. Secondly, several other changes were also undertaken to facilitate the construction of an adequate situational model.

Three dependent measures were taken: 1) free recall, in which we distinguished two levels, high-and low-level idea-units. 2) Subjects had to write the 7-8 most important ideas in the text. 3) A comprehension and learning test in which subjects answered five open questions measuring their skills in applying textual information to new contexts.

Separate ANOVA's were carried out for each dependent measurement. Recall of high-level idea units was found to be significantly different with respect to the text and strategies used, and between the two groups of undergraduates with different prior knowledge. Post-hoc analyses showed significant differences between groups, following the order: UPh > UPs > $\mathrm{SS}$ and $\mathrm{SnS}$. In addition, the groups who read the adapted passage recalled significantly more information than those who read the original.

Results from the comprehension and learning task showed that when variables were considered separately, significant differences were found in all of them. There was also a significant interaction effect between text and strategies variables. This is explained by the dramatic improvement in the scores of secondary school students, particularly science students, who read the adapted passage; which did not occur with the undergraduates. In relation to understanding the main ideas in a text, significant differences were again found for text and strategies variables. Post-hoc analyses produced significant differences both between the two groups of undergraduate stu- 
dents who read the adapted version and between those reading the original, though this did not occur with the secondary school students.

To summarize, the results generally support our first hypothesis, as text manipulations had a positive effect on all dependent measures, though there were some between tasks differences. Results also support the second hypothesis, as the strategies used were found to play an important role on recall. It seems, nevertheless, that macrostrategies are more important for recall than previous knowledge, at least under certain circumstances. The third hypothesis is partially confirmed, as previous knowledge was found to play an important role in learning tasks, though this is limited due to the interaction found between learning and type of text. Finally the forth hypothesis is generally confirmed. The variable strategies produced significant differences between groups in understanding the main ideas in a text, undergraduates scoring higher than secondary school students.

The study explains the relationships established between the three groups of variables studied. It also lends support to van Dijk and Kintsch's distinction of the two types of mental representations formed as a result of text processing. Finally, it shows how to elaborate text that facilitate learning. There are still, however, several issues that remain to be clarified in future studies such as the differential effect of the two types of text modifications on the text-base and the situational model; or analysing in greater detailed the influence of strategies and prior knowledge on learning and recall, which requires more precise and diversified measurements. 